\title{
Finite Element Schemes for Fermi Equation
}

\author{
M. Asadzadeh ${ }^{1, b)}$, L. Beilina ${ }^{1, \mathrm{c})}$, M. Naseer ${ }^{1, \mathrm{~d})}$ and C. Standar ${ }^{1, \mathrm{a})}$ \\ ${ }^{1}$ Department of Mathematical Sciences, Chalmers University of Technology and Gothenburg University, \\ 41296 Gothenburg, Sweden \\ a)Corresponding author: standarc@chalmers.se \\ b)mohammad@chalmers.se \\ c) larisa@chalmers.se \\ d)na.seer@hotmail.com
}

Abstract. A priori error estimates are derived for the streamline diffusion (SD) finite element methods for the Fermi pencil-beam equation. Two-dimensional numerical examples confirm our theoretical investigations.

\section{Introduction}

The Boltzmann transport equation modeling the energy independent pencil beam process can be written as a two-point boundary value problem viz,

$$
\mu \frac{\partial u}{\partial x}+\eta \frac{\partial u}{\partial y}+\xi \frac{\partial u}{\partial z}=\int_{S^{2}} \sigma_{s}\left(\mathbf{v} \cdot \mathbf{v}^{\prime}\right)\left[u\left(\mathbf{x}, \mathbf{v}^{\prime}\right)-u(\mathbf{x}, \mathbf{v})\right] d^{2} \mathbf{v}^{\prime}, \quad 0<x<1
$$

where $\mathbf{x}=(x, y, z)$ and $\mathbf{v}=(\mu, \eta, \xi)$ are the space and velocity vectors, respectively. The model problem concerns sharply forward peaked beam of particles entering the spatial domain at $x=0$. Fermi proposed the following form of, projected, Fokker-Planck model of (1):

$$
\frac{\partial u}{\partial x}+\eta \frac{\partial u}{\partial y}+\xi \frac{\partial u}{\partial z}=\frac{\sigma_{t r}}{2}\left(\frac{\partial^{2}}{\partial \eta^{2}}+\frac{\partial^{2}}{\partial \xi^{2}}\right) u(\mathbf{x}, \mathbf{v}), \quad 0<x<1,
$$

with

$$
u(0, y, z, \eta, \xi)=\delta(y) \delta(z) \delta(\eta) \delta(\xi)
$$

Extending $(\eta, \xi)$ to $\mathbb{R}^{2}$, the Fourier transformation with respect to $y, z, \eta$, and $\xi$, assuming constant $\sigma_{t r}$, yields the following exact solution for the angular flux

$$
u(x, y, z, \eta, \xi)=\frac{3}{\pi^{2} \sigma_{t r}^{2} x^{4}} \exp \left[-\frac{2}{\sigma_{t r}}\left(\frac{\eta^{2}+\xi^{2}}{x}-3 \frac{y \eta+z \xi}{x^{2}}+3 \frac{y^{2}+z^{2}}{x^{3}}\right)\right] .
$$

The closed form solution (4) was first derived by Fermi as referred in [5]. 
Restricted to bounded phase-space domain, equation (2) can be written as

$$
\begin{cases}u_{x}+\mathbf{v} \cdot \nabla_{\perp} u=\frac{\sigma_{t r}}{2} \Delta_{\mathbf{v}} u & \text { in } \quad \Omega:=\Omega_{\mathbf{x}} \times \Omega_{\mathbf{v}}, \\ \nabla_{\mathbf{v}} u\left(x, x_{\perp}, \mathbf{v}\right)=0 & \text { for } \quad\left(x, x_{\perp}, \mathbf{v}\right) \in \Omega_{\mathbf{x}} \times \partial \Omega_{\mathbf{v}}, \\ u\left(0, x_{\perp}, \mathbf{v}\right)=u_{0}\left(x_{\perp}, \mathbf{v}\right) & \text { for } \quad\left(x_{\perp}, \mathbf{v}\right) \in \Omega_{x_{\perp}} \times \Omega_{\mathbf{v}}=: \Omega_{\perp}, \\ u\left(x, x_{\perp}, \mathbf{v}\right)=0 & \text { on } \quad \Gamma_{\tilde{\beta}}^{-} \backslash\left\{\left(0, x_{\perp}, \mathbf{v}\right)\right\},\end{cases}
$$

where $\mathbf{v}=(\eta, \xi), \nabla_{\perp}=(\partial / \partial y, \partial / \partial z)$ and

$$
\Gamma_{\tilde{\beta}}^{-}:=\left\{\left(x, x_{\perp}, \mathbf{v}\right) \in \partial \Omega, \mathbf{n} \cdot \tilde{\beta}<0\right\}
$$

is the inflow boundary with respect to $\tilde{\beta}:=(1, \mathbf{v}, 0,0)$ and $\mathbf{n}$ is the outward unit normal to the boundary $\partial \Omega$.

\section{The model problem}

We consider now a forward peaked narrow radiation beam entering into the symmetric domain $I_{y} \times I_{\eta}=$ $\left[-y_{0}, y_{0}\right] \times\left[-\eta_{0}, \eta_{0}\right] ;\left(y_{0}, \eta_{0}\right) \in \mathbb{R}_{+}^{2}$ at $(0,0)$ and penetrating in the direction of the positive $x$-axis. Then the computational domain $\Omega$ is a three dimensional slab with $(x, y, \eta) \in \Omega=I_{x} \times I_{y} \times I_{\eta}$ where $I_{x}=[0, L]$. In this way, the problem (5) will be transformed into the following lower dimensional model problem

$$
\left\{\begin{aligned}
u_{x}+\eta u_{y}=\frac{1}{2} \sigma_{t r} u_{\eta \eta} & (x, y, \eta) \in \Omega \\
u_{\eta}\left(x, y, \pm \eta_{0}\right)=0 & (x, y) \in I_{x} \times I_{y} \\
u(0, y, \eta)=f(y, \eta) & (y, \eta) \in I_{y} \times I_{\eta} \\
u(x, y, \eta)=0 & \text { on } \Gamma_{\beta}^{-} \backslash\{(0, y, \eta)\}
\end{aligned}\right.
$$

For (7) we implement two different versions of the SD method: the semi-streamline diffusion and the characteristic streamline diffusion. Both cases are discretized using linear polynomials.

\section{The semi-streamline diffusion method}

In this version we derive a discrete scheme for computing the approximate solution $u_{h}$ of the exact solution $u$ using the SD-method for discretizing the $(y, \eta)$-variables combined with the backward Euler method for the $x$-variable. We start by introducing the bilinear forms $a(\cdot, \cdot)$ and $b(\cdot, \cdot)$ for the problem (7) as:

$$
\begin{aligned}
a(u, w)= & \left(\eta u_{y}, w\right)_{\perp}+\delta\left(\eta u_{y}, \eta w_{y}\right)_{\perp}+\frac{1}{2}\left(\sigma_{t r} u_{\eta}, w_{\eta}\right)_{\perp} \\
& +\frac{1}{2} \delta\left(\sigma_{t r} u_{\eta}, w_{y}+\eta w_{y \eta}\right)_{\perp}-\left.\frac{1}{2} \delta \int_{I_{y}} \sigma_{t r} \eta u_{\eta} w_{y}\right|_{\eta=-\eta_{0}} ^{\eta=\eta_{0}} d y, \\
b(u, w)= & (u, w)_{\perp}+\delta\left(u, \eta w_{y}\right)_{\perp}, \quad(\cdot, \cdot)_{\perp}:=(\cdot, \cdot)_{I_{y} \times I_{\eta}} .
\end{aligned}
$$

The continuous problem: for each $x \in(0, L]$, find $u(x, \cdot) \in H_{\beta}^{1}$ such that

$$
b\left(u_{x}, w\right)+a(u, w)=0, \quad \forall w \in H_{\beta}^{1},
$$

where

$$
H_{\beta}^{1}:=\left\{w \in H^{1}\left(I_{y} \times I_{\eta}\right) ; w=0 \text { on } \Gamma_{\beta}^{-}\right\},
$$

and

$$
\Gamma_{\beta}^{-}:=\left\{(y, \eta) \in \Gamma:=\partial\left(I_{y} \times I_{\eta}\right), \text { with } \mathbf{n} \cdot \beta<0\right\},
$$


with $\beta=(\eta, 0)$. Then the semi-streamline diffusion method for the continuous problem (7) reads as follows: for each $x \in(0, L]$, find $u_{h}(x, \cdot) \in \mathcal{V}_{h, \beta}$ such that,

$$
b\left(u_{h, x}, w\right)+a\left(u_{h}, w\right)=0, \quad \forall w \in \mathcal{V}_{h, \beta},
$$

where $\mathcal{V}_{h, \beta} \subset H_{\beta}^{1}$ consists of continuous piecewise linear functions. Writing the discrete solution as

$$
u_{h}(x, y, \eta)=\sum_{j=1}^{N} U_{j}(x) \phi_{j}(y, \eta)
$$

where $N$ is the number of nodes in the mesh, and inserting (11) into (10) with $w=\phi_{i}$ for $i=1,2, \ldots, N$ we get the following discrete system of equations in a matrix form

$$
\left[B+k_{m} A\right] U^{m+1}=B U^{m},
$$

where $m$ is the number of iteration in a backward Euler's scheme. Here, $U=\left[U_{1}, \ldots, U_{N}\right]^{T}, B=\left(b_{i j}\right), b_{i j}=$ $b\left(\phi_{j}, \phi_{i}\right)$ and $A=\left(a_{i j}\right), a_{i j}=a\left(\phi_{j}, \phi_{i}\right), i, j=1,2, \ldots, N$.

\section{Characteristic Streamline Diffusion Method}

In this part we construct an oriented phase-space mesh to obtain the characteristic streamline diffusion method. Here, we need to construct a new subdivision of $\Omega=I_{x} \times I_{y} \times I_{\eta}$. To this end and for $m=1,2, \ldots, M$, we define a subdivision of $\Omega_{m}=\left[x_{m-1}, x_{m}\right] \times I_{y} \times I_{\eta}:=I_{m} \times I_{y} \times I_{\eta}$ into elements

$$
\hat{\tau}_{m}=\left\{\left(x, y+\left(x-x_{m}\right) \eta, \eta\right):(y, \eta) \in \tau \in \mathcal{T}_{h}, x \in I_{m}\right\}
$$

where $\mathcal{T}_{h}$ is a previous triangulation of $I_{\perp}$. Then we introduce, slabwise, the function spaces

$$
\hat{\mathcal{V}}_{m}=\left\{\hat{w} \in C\left(\Omega_{m}\right): \hat{w}(x, y, \eta)=w\left(y+\left(x-x_{m}\right) \eta, \eta\right), w \in \mathcal{V}_{h, \beta}\right\} .
$$

In other words $\hat{\mathcal{V}}_{m}$ consists of continuous functions $\hat{w}(x, y, \eta)$ on $\Omega_{m}$ such that $\hat{w}$ is constant along characteristics $(\hat{y}, \hat{\eta})=(y+x \eta, \eta)$ parallel to the sides of the elements $\hat{\tau}_{m}$, meaning that the derivative in the characteristic direction: $\hat{w}_{x}+\eta \hat{w}_{y}=0$. The SD method can now be reduced to the following formulation (where only the $\sigma_{t r}$-term survives): find $\hat{u}_{h}$ such that, for each $m=1,2, \ldots, M,\left.\hat{u}_{h}\right|_{\Omega_{m}} \in \hat{\mathcal{V}}_{m}$

$$
\begin{array}{r}
\frac{1}{2} \int_{\Omega_{m}} \sigma_{t r} \hat{u}_{h, \eta} w_{\eta} d x d y d \eta+\int_{I_{\perp}} \hat{u}_{h,+}\left(x_{m-1}, y, \eta\right) w_{+}\left(x_{m-1}, y, \eta\right) d y d \eta \\
=\int_{I_{\perp}} \hat{u}_{h,-}\left(x_{m-1}, y, \eta\right) w_{+}\left(x_{m-1}, y, \eta\right) d y d \eta, \quad \forall w \in \hat{\mathcal{V}}_{m}
\end{array}
$$

\section{Numerical examples}

In this section we will demonstrate the performance of an adaptive finite element method for the solution of the model problem (7) using module LIVE LINK MATLAB in Matlab COMSOL Multiphysics. The computational domain $\Omega_{\perp}=I_{y} \times I_{\eta}$ is chosen as $\Omega_{\perp}=\{(y, \eta) \in(-1.0,1.0) \times(-1.0,1.0)\}$. We have performed tests with a fixed diffusion coefficient $\sigma_{t r}=0.002$ and used the backward Euler scheme (12) with the time step $k_{m}=0.01$. Note that [2] shows oscillatory behavior of the solution $u_{h}$ when the semi-streamline diffusion method was used, and layer behavior when the standard FEM was applied to solve the problem (7). Our numerical tests show significant improvement of results of [2] since we have applied adaptive finite 
TABLE 1. Absolute $e_{n}=\left\|u-u_{h}^{n}\right\|_{L_{2}\left(\Omega_{\perp}\right)}$ and relatives $e_{n} / e_{n+1}$ errors on the adaptively refined meshes. Here, $u_{h}^{n}$ is computed using semi-streamline diffusion method with $\widetilde{\gamma}=0.5$ in the adaptive algorithm, see [3] for details.

\begin{tabular}{l|lllll}
\hline $\begin{array}{l}\text { Nr. of } \\
\text { refinement, } n\end{array}$ & $\begin{array}{l}\text { Nr. of } \\
\text { elements }\end{array}$ & $\begin{array}{l}\text { Nr. of } \\
\text { vertices }\end{array}$ & DOF & $e_{n}=\left\|u-u_{h}^{n}\right\|_{L_{2}}$ & $e_{n} / e_{n+1}$ \\
\hline 0 & 272 & 157 & 1285 & $1.565 \mathrm{e}-05$ & \\
1 & 1271 & 597 & 5115 & $9.732 \mathrm{e}-07$ & 16.08 \\
2 & 5084 & 2267 & 20937 & $6.052 \mathrm{e}-08$ & 16.08 \\
3 & 20336 & 9075 & 79825 & $3.771 \mathrm{e}-09$ & 16.05 \\
\hline
\end{tabular}

TABLE 2. Absolute $e_{n}=\left\|u-u_{h}^{n}\right\|_{L_{2}\left(\Omega_{\perp}\right)}$ and relative $e_{n} / e_{n+1}$ errors on the adaptively refined meshes. Here, $u_{h}^{n}$ is computed using semi-streamline diffusion method with $\widetilde{\gamma}=0.7$ in the adaptive algorithm, see [3] for details.

\begin{tabular}{l|lllll}
\hline $\begin{array}{l}\text { Nr. of } \\
\text { refinement, } n\end{array}$ & $\begin{array}{l}\text { Nr. of } \\
\text { elements }\end{array}$ & $\begin{array}{l}\text { Nr. of } \\
\text { vertices }\end{array}$ & DOF & $e_{n}=\left\|u-u_{h}^{n}\right\|_{L_{2}}$ & $e_{n} / e_{n+1}$ \\
\hline 0 & 272 & 157 & 1285 & $1.565 \mathrm{e}-05$ & \\
1 & 1088 & 585 & 5017 & $1.484 \mathrm{e}-06$ & 15.98 \\
2 & 4352 & 2257 & 19825 & $9.289 \mathrm{e}-07$ & 16.02 \\
3 & 17408 & 8865 & 78817 & $5.799 \mathrm{e}-08$ & 16.02 \\
\hline
\end{tabular}

element algorithm for computations, see [3] for details of this algorithm and additional numerical tests. We have compared our computations with the analytic solution for the problem (7) given by

$$
u(x, y, \eta)=\frac{\sqrt{3}}{\pi \sigma_{t r} x^{2}} \exp \left[-\frac{2}{\sigma_{t r}}\left(\frac{3 y^{2}}{x^{3}}-\frac{3 y \eta}{x^{2}}+\frac{\eta^{2}}{x}\right)\right],
$$

when the initial data is given by $u(0, y, \eta)=\delta(y) \delta(\eta)$. Tables show results of our computations on a local adaptivelly refined meshes when we solved the problem (7) with a "hyperbolic type" initial condition

$$
u(0, y, \eta)=f(y, \eta)=\frac{1}{\sqrt{y^{2}+\eta^{2}+\alpha}}, \quad(y, \eta) \in \Omega_{\perp}, \quad \alpha=0.19 .
$$

\section{REFERENCES}

[1] M. Asadzadeh, Streamline diffusion methods for Fermi and Fokker-Planck equations, Transport Theory Statistica Physics, 26(3), pp. 319-340, 1997.

[2] M. Asadzadeh and E. Larsen, Linear transport equations in flatland with small angular diffusion and their finite element approximation, Mathematical and Computer Modelling, Elsevier, pp. 495 $-514,2007$.

[3] M. Asadzadeh, L. Beilina, M. Naseer, C. Standar, A priori error estimates and computational studies for a Fermi pencil-beam equation, arXiv:1606.05085, 2016.

[4] M. Asadzadeh, On the stability of characteristic schemes for the fermi equation, Appl. Comput. Math., 1(1), pp.158-174, 2002.

[5] B. Rossi, K. Greisen, Cosmic-ray theory, Rev. Mod. Phys., 13, pp. 309-340, 